\title{
Estruturas mistas em madeira-concreto: uma técnica racional para pontes de estradas vicinais
}

\author{
Timber-concrete composite structures: a rational technique for bridges of vicinal roads
}

\author{
Julio Soriano ${ }^{\mathrm{I}}$ Nilson Tadeu Mascia ${ }^{\mathrm{II}}$
}

\section{- REVISÃO BIBLIOGRÁFICA -}

\section{RESUMO}

De modo geral, a aplicação racional de materiais de construção requer o desenvolvimento ou aprimoramento de técnicas construtivas. Nesse sentido, a utilização do concreto e da madeira, materiais tradicionais e de grande aceitação em construções rurais, formando estruturas mistas, constitui-se em uma solução viável por apresentar desempenhos estrutural $e$ econômico adequados, bem como boa durabilidade. Particularmente, a efetivação desse sistema estrutural deve ser assegurada por meio de uma ligação na interface dos materiais, podendo ser do tipo discreta (elementos metálicos: parafusos, barras de aço, pregos, etc.) ou contínua (adesivo epóxi). $\mathrm{Na}$ construção civil, diversos tipos de aplicações podem ser contemplados com esse sistema estrutural, tais como restaurações ou construções de novas pontes. De fato, em estradas vicinais, existe um grande número de pontes de madeira que necessitam de reformas e também há a necessidade de construção de pontes novas. De maneira geral, as pontes são expostas a condições severas de intemperismo e carregamento, necessitando, desse modo, de estudos cuidadosos para que se possa avaliar com maior precisão os mecanismos da ligação, bem como o comportamento da estrutura mista em toda sua vida útil. Nesse contexto, ao serem analisados, neste artigo, os diversos aspectos que envolvem o desempenho das estruturas mistas em madeira-concreto e também o comportamento mecânico das ligações nessas estruturas, podese concluir que essa técnica é viável para construção de pontes das estradas vicinais. Além disso, pesquisas adicionais sobre efeitos de carregamento de longa duração, variações de temperatura e umidade são necessárias para melhorar os modelos de simulações.

Palavras-chave: sistema estrutural, ligação, cisalhamento horizontal, durabilidade, painéis mistos.

\begin{abstract}
In general, the rational application of construction materials requires the development or improvement of building techniques. Thus, the use of concrete and timber, traditional materials largely accepted in rural buildings, making composite structures, constitutes a possible solution for presenting adequate structural and economical performances and durability too. In particular, the effectiveness of this structural system must be assured by a connection at the interface of the materials, and it can be of discrete (metallic elements: screws, steel bars, nails etc) or continuous types (epoxy adhesive). Several kinds of civil construction applications can be contemplated by this structural system, as in restorations or construction of new bridges. In fact, along the vicinal roads, there is a large number of timber bridges that need restorations and there is a need to construct new bridges as well. General speaking, bridges are exposed to severe weather and loading conditions, and, thus, they must be carefully studied, so that the connection mechanisms can be more precisely evaluated, as well as the composite structure behavior during its entire life. In this context, by analyzing the several aspects that involve the performance of the timber-concrete composite structures and, also the mechanical behavior of the connections in these structures, we conclude, in this article, that this construction technique is feasible for bridges in vicinal roads. Further, additional research on the long-term loading effects, temperature and moisture variations, is necessary to improve the simulation models.
\end{abstract}

Key words: structural system, connection system, horizontal shear, durability, composite panel.

\footnotetext{
'Departamento de Construções Rurais e Ambiência, Faculdade de Engenharia Agrícola, Universidade Estadual de Campinas (Unicamp), CP 6011, 13083-875, Campinas, SP, Brasil. E-mail: julio.soriano@feagri.unicamp.br. Autor para correspondência.

IIDepartamento de Estruturas, Faculdade de Engenharia Civil, Unicamp, Campinas, SP, Brasil.
} 


\section{INTRODUÇÃO}

As pontes podem ser definidas como obrasde-arte destinadas à transposição de obstáculos naturais ou não, visando à locomoção de veículos diversos e pedestres com segurança e conforto. Nas estradas vicinais, essas obras são de fundamental importância para integração regional e, do ponto de vista do setor do agronegócio, para proporcionar condições para o tráfego de caminhões e máquinas durante a safra, bem como no escoamento final da produção agrícola.

Grande parte das pontes de estradas vicinais é executada em madeira e, embora tais estruturas representem baixo custo de execução, podem, conforme MASCIA \& SORIANO (2004), apresentar uma pequena durabilidade e um baixo desempenho estrutural. Dentre os materiais de uso comum na construção de estruturas rurais, destaca-se a madeira que do ponto de vista ambiental constitui-se num recurso renovável e que requer baixo consumo energético para a sua produção (STEINBERG et al., 2003; GUTKOWSKI et al., 2004). A madeira, conjuntamente com o concreto, pode constituir uma seção mista, resultando em diversas vantagens relacionadas, por exemplo, à resistência e à durabilidade da construção, conforme descrevem GELFI et al. (2002) e MIOTTO \& DIAS (2006).

Em geral, utilizam-se nessas estruturas mistas sistemas de ligação formados por elementos metálicos dispostos ao longo da superfície de contato madeira-concreto, que devem influenciar o comportamento desse tipo de sistema estrutural. Em razão das propriedades diferentes dos três materiais envolvidos, há grande dificuldade de se prever o mecanismo de trabalho exato do sistema de ligação (DIAS et al., 2007a). Em razão das incertezas relacionadas ao módulo de deslizamento, pesquisas são realizadas a fim de comparar resultados experimentais com resultados de simulações.

A presente revisão bibliográfica tem por objetivo apresentar aspectos favoráveis de desempenho estrutural e durabilidade, proporcionados pelo método das estruturas mistas em madeira-concreto para construção de pontes de estradas vicinais.

Horizontes para aplicações da técnica de estrutura mista Um elevado número de pontes de estradas vicinais no Brasil foi construído com madeira, havendo em grande parte ausência de projetos, programas de inspeções e medidas preventivas que assegurassem uma longa vida útil dessas obras. O baixo desempenho estrutural dessas obras-de-arte, bem como o comprometimento da durabilidade, pode estar associado a técnicas construtivas inadequadas. Conforme CLOUSTON et al. (2005), as pontes rodoviárias e passarelas construídas somente em madeira estão sujeitas à deterioração mais acelerada pelas ações de uso e ambiental. MASCIA \& SORIANO (2004) descrevem que algumas pontes recebem uma camada de solo sobre o tabuleiro de madeira, conforme ilustrado nas figuras $1 \mathrm{~A}$ e $1 \mathrm{~B}$. Se, por um lado, essa camada de solo reduz o impacto ou atrito sobre a estrutura, por outro, representa um fator determinante para as condições da biodeterioração da madeira. Observa-se ainda que essa camada de solo eleva a ação permanente sobre a construção.

No sistema de estradas paulistas, estima-se um total de $1100 \mathrm{~km}$ de pontes vicinais, conforme CALIL JÚNIOR et al. (2006), das quais muitas necessitam de procedimentos imediatos de recuperação ou adequação. Ressalta-se ainda a necessidade de construção de novas pontes, que são de extrema importância para a infra-estrutura de transporte.

A madeira desempenhou valor histórico no desenvolvimento da humanidade e, graças à proximidade das fontes desse material natural e a sua fácil trabalhabilidade é ainda amplamente utilizada nas construções rurais. A produção e o processamento de madeira de reflorestamento, tais como pinus e eucalipto, são atrativos para o setor do agronegócio. No âmbito das grandes construções, o eucalipto, mais recentemente, tem sido empregado em pontes e passarelas, como ilustrado por CALIL JÚNIOR et al. (2006).

Com elevada sensibilidade às variáveis ambientais, tais como temperatura e umidade relativa do ar, a madeira é um material suscetível às alterações dos seus estados de deformação e tensão (FRAGIACOMO, 2006). O emprego de peças de madeira muitas vezes é limitado pelas dimensões das seções comercias, bem como pelo seu baixo módulo de elasticidade. No entanto, do ponto de vista tecnológico, a Madeira Laminada Colada (MLC) proporciona seções transversais maiores que aquelas comumente encontradas na madeira serrada, bem como as seções em forma de arco com efeitos favoráveis às pontes (STOJIC \& KAJGANOVIC, 2007). Além disso as lâminas selecionadas são previamente secas em estufas e submetidas ao processo de tratamento preservativo, assegurando qualidade e durabilidade à construção. Quanto às técnicas de uso estrutural da madeira, um sistema de placa formado por peças protendidas transversalmente é proposto como uma solução viável para as pontes de estradas rurais (FONTE \& CALIL JÚNIOR, 2007). Do ponto de vista de durabilidade, há 


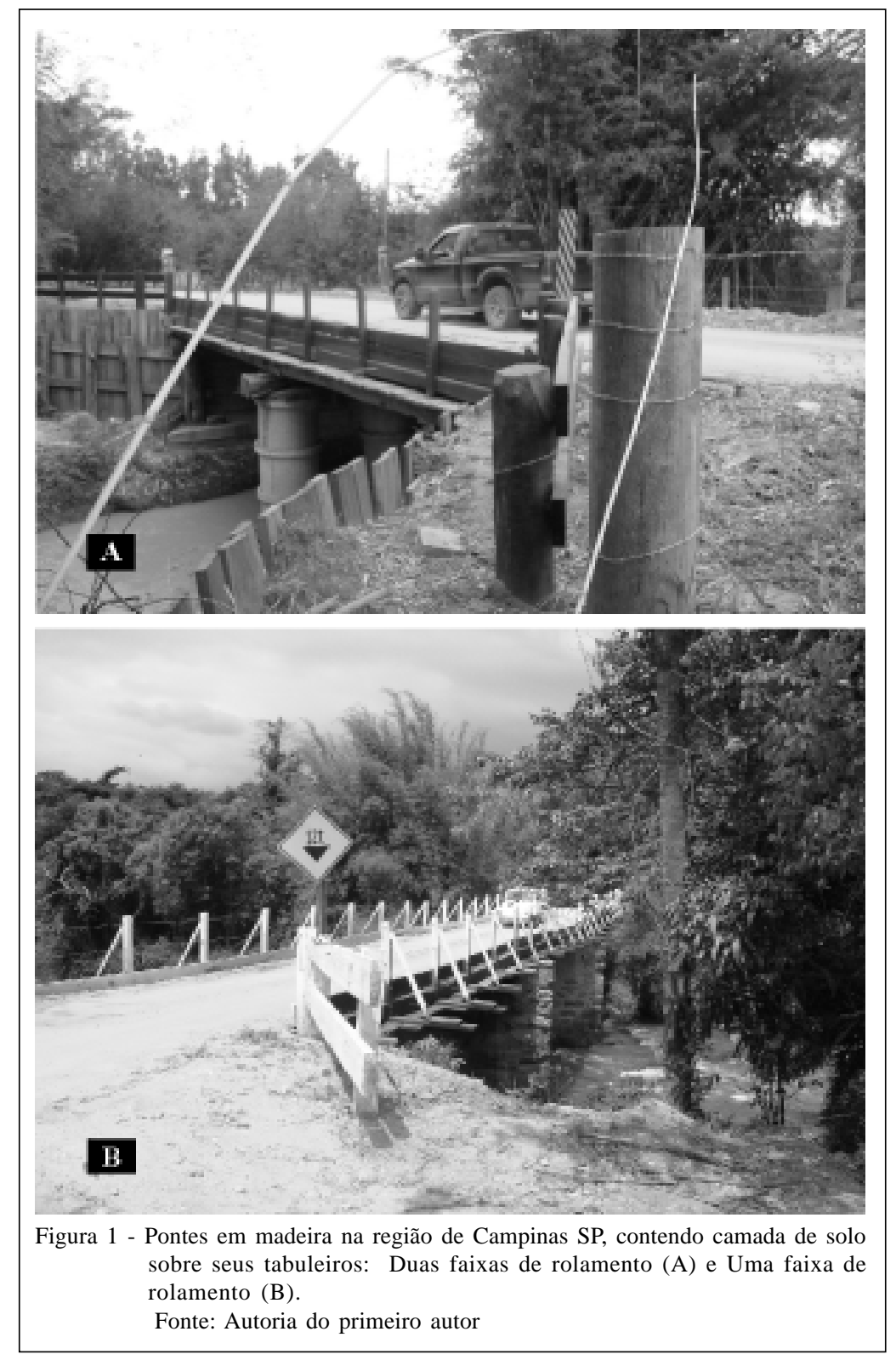

necessidade de capeamento dessa placa com pavimentação asfáltica ou com concreto, por exemplo.

O concreto armado oferece uma grande versatilidade para se moldar as diversas formas arquitetônicas, conforme CARVALHO \& FIGUEIREDO FILHO (2004); porém, apresenta um elevado peso específico e uma alta condutividade térmica, principalmente, se comparado às espécies de madeira de baixa densidade. Quando não pré-fabricado, em geral, ele requer um grande consumo de fôrmas e escoras. É um pseudo-sólido sujeito às diversas ações patológicas extrínsecas e intrínsecas (SOUZA \& RIPPER, 2004). Por isso, para se assegurar a durabilidade da estrutura de concreto armado, em razão da classe de agressividade ambiental, a ASSOCIAÇÃO BRASILEIRA DE NORMAS TÉCNICAS - ABNT (6118:2003) prescreve valores mínimos para a resistência característica à compressão e ao cobrimento nominal da armadura, bem como estabelece valores máximos para relação água/cimento.

A preocupação do emprego racional dos materiais: madeira, concreto e aço é um tanto remota, porém tem se firmado mais recentemente devido à escassez dos materiais, da necessidade de redução dos desperdícios na construção e da preservação ambiental, dentre outras razões. Por esses aspectos, a seção mista em madeira-concreto é apresentada como solução viável.

Ciência Rural, v.39, n.4, jul, 2009. 
A seção mista consiste basicamente de uma placa em concreto conectada a peças de madeira (Figura 2), por meio de ligações discretas, como, por exemplo: entalhes na madeira, parafusos, pregos, tarugos de aço ou, ainda, conectores metálicos especiais (CECCOTTI, 1995; GUTKOWSKI et al., 2004; FRAGIACOMO et al., 2007). O sistema ligação contínuo pode ser obtido, por exemplo, com aplicações de adesivo epóxi (STOJIC \& CVETKOVIC, 2001; BRUNNER et al., 2007). A escolha dentre os diversos dispositivos está atrelada às condições de projeto, tais como: carregamento, rigidez desejada para a estrutura, custos e exeqüibilidade da ligação.

O sistema de ligação deve garantir a transferência dos esforços de cisalhamento horizontal, influenciando diretamente a distribuição de forças internas e as deformações da estrutura, bem como o afastamento vertical entre o concreto e a madeira, conforme GIRHAMMAR \& GOPU (1993) e FRAGIACOMO \& CECCOTTI (2006). A ligação deve ser concebida de maneira que os materiais sejam aproveitados com suas melhores propriedades mecânicas, isto é, o concreto solicitado à compressão e a madeira solicitada à tração (AHMADI \& SAKA, 1993; KAVALIAUSKAS et al., 2005; BRUNNER et al., 2007). Tal premissa fundamenta o princípio básico da otimização, ou seja, uma construção mais leve, porém com elevado desempenho estrutural.

A viabilidade de utilização da técnica de estruturas mistas pode ser vista por diversos aspectos em relação aos sistemas das estruturas convencionais constituídas somente em madeira ou concreto armado. Em relação às construções somente em madeira, CLOUSTON et al. (2005) e MIOTTO \& DIAS (2006) destacam que as estruturas mistas apresentam comportamento mais adequado de resistência à propagação do fogo, com melhores propriedades acústicas e de vibrações. Nessa comparação, STOJIC \& CVETKOVIC (2001) e STOJIC \& KAJGANOVIC (2007) destacam também uma maior rigidez da seção transversal, bem como a maior resistência e estabilidade aos efeitos sísmicos. Pode-se concluir que essas características positivas resultam da incorporação da massa do concreto à seção da estrutura, sendo imprescindível o sistema de ligação.

Já, quando comparadas às estruturas em concreto armado, as estruturas mistas representam uma redução de custo direto por área, um menor consumo de material para fôrmas e, em geral, dispensam o uso de escoras para concretagem (CECCOTTI, 1995; GUTKOWSKI et al., 2004). A redução da ação permanente resulta também em elementos de fundações mais simples (STOJIC \& CVETKOVIC, 2001). Adicionalmente, como parte da estrutura é em madeira, haverá uma redução do tempo de execução da construção e será ainda menor em situações que se utilizem painéis pré-fabricados.

Pesquisas para aplicações de estruturas mistas em madeira-concreto em pontes foram iniciadas nos Estados Unidos da América, na década de 1930. Na Europa, por exemplo, essa técnica passou a ser explorada efetivamente a partir da década de 1980 (JUTILA et al., 1997). Atualmente, atenta-se para a necessidade de difusão da técnica, justamente pelas limitações dos recursos naturais e, também, pela busca de maior durabilidade das construções.

Além das aplicações em pontes, as estruturas mistas em madeira-concreto são adequadas às outras modalidades de construção, por exemplo: residências, escolas e comércios, sendo destacadas para restaurações de pisos e forros de construções antigas (CECCOTTI, 1995; GELFI et al., 2002). Além dos benefícios de desempenhos estruturais, MIOTTO \& DIAS (2006) enfatizam a importância dessas

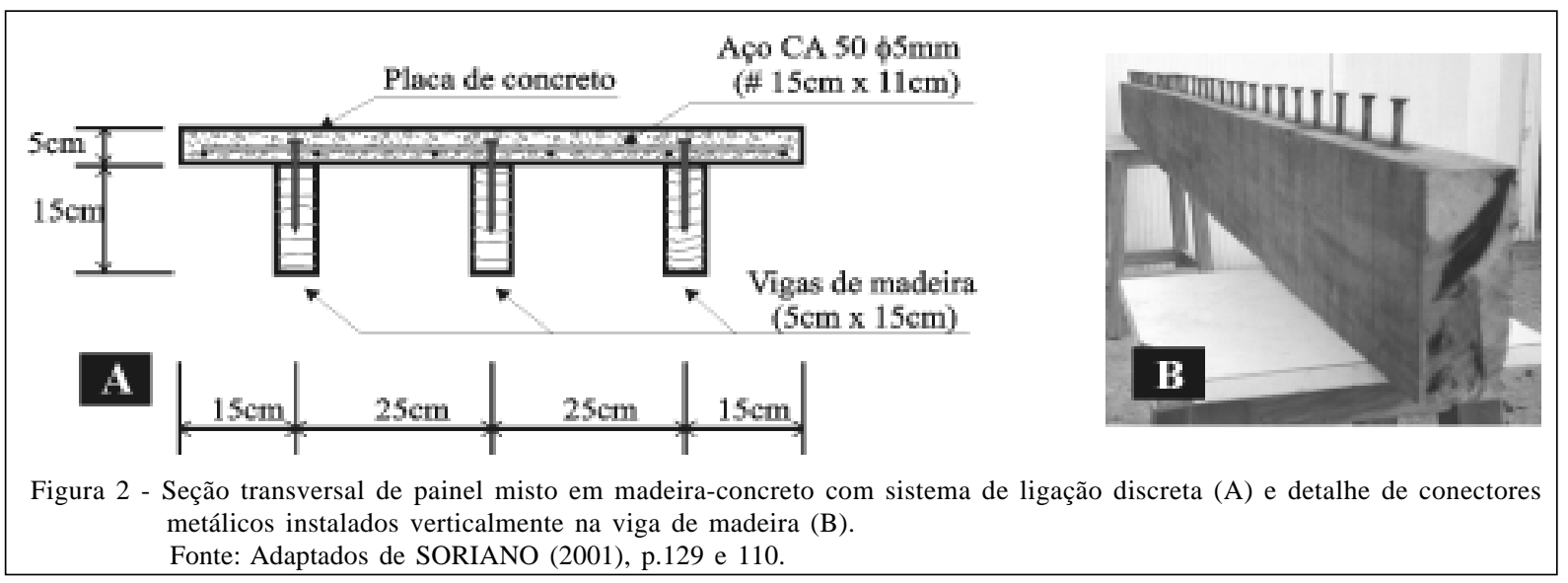

Ciência Rural, v.39, n.4, jul, 2009. 
aplicações na preservação de pisos de prédios históricos, sendo mantidas as características arquitetônicas da construção.

\section{Sistema de ligação}

O sistema de ligação que deve ser empregado na interface de contato madeira-concreto, dependendo de suas características, pode elevar a capacidade de carregamento da estrutura da ordem de duas vezes, se comparado a uma seção com os materiais que trabalham independentemente, conforme descrevem AHMADI \& SAKA(1993). Já a rigidez pode ser elevada de três a quatro vezes em relação a uma estrutura fabricada exclusivamente em madeira (CECCOTTI, 1995). Esta última característica implica deslocamentos menores, uma vez que as flechas são inversamente proporcionais à rigidez da estrutura.

No entanto, o sistema de ligação caracteriza o comportamento da estrutura mista, podendo ser classificado como rígido ou flexível, sendo a primeira forma a de maior eficiência, desde que o deslizamento de interface seja desprezível ou inexistente, representando uma composição total. Na segunda forma, a eficiência da ligação é relativa ao tipo de conector empregado. A análise experimental de um modelo de estrutura para ponte em placa de concreto e toras de eucalipto, cujo sistema de ligação era formado por barras com 12,5mm de diâmetro, de aço CA50, fixadas verticalmente na madeira, revelou eficiência do sistema de aproximadamente 80\% (SEGUNDINHO \& MATTHIESEN, 2004).

Num conjunto de resultados de ensaios de painéis mistos em madeira e concreto, com $80 \mathrm{~cm}$ de largura e $300 \mathrm{~cm}$ de vão, com seção transversal representada na figura 2A, SORIANO (2001) comprovou o desempenho estrutural graças às ligações por pregos (diâmetro de 6,58mm) espaçados a cada $5 \mathrm{~cm}$ e parafusos (diâmetro de 12,70mm) espaçados a cada $7,5 \mathrm{~cm}$. As nervuras de cada painel foram constituídas por três vigas de madeira da espécie Cupiúba (Goupia glabra), com seções transversais de $5 \mathrm{~cm}$ x 15cm (Resistência média à compressão na direção paralela às fibras igual 60,57MPa e Módulo de elasticidade na direção paralela às fibras igual $14700 \mathrm{MPa}$, valores esses referentes ao teor de umidade 11,46\%). Na mesa de cada painel, utilizou-se concreto usinado (resistência média à compressão igual 22,05MPa e módulo de elasticidade igual 19297MPa), contendo uma malha de aço CA50, formada por barras de $5 \mathrm{~mm}$ de diâmetro. Na figura 3, observam-se os respectivos valores experimentais e teóricos, sendo para estes últimos supostas as situações de inexistência de ligação e a existência de ligação perfeitamente rígida (composição total). Para o nível de deslocamento de $15 \mathrm{~mm}$, que representa o Estado Limite de Serviço, as ligações proporcionaram acréscimos de cargas de aproximadamente 50\% e 100\%, para as ligações com pregos e parafusos, respectivamente. Nota-se que a eficiência do segundo sistema, cuja rigidez à flexão de cada elemento de conexão é maior, mostrou-se mais condizente com as informações apresentadas por outros autores.

No sentido de se obterem sistemas de ligações mais eficientes e de baixo custo, diversas pesquisas têm sido realizadas. Pode-se quantificar a eficiência de uma ligação por meio do módulo de deslizamento (razão entre Força e Deslizamento), obtido experimentalmente em corpos-de-prova representativos da ligação. STOJIC \& CVETKOVIC (2006) e DIAS et al. (2007a) ressaltam a importância desses ensaios, cujos resultados são dependentes de diversas variáveis, tais como: módulos de elasticidade e resistências da madeira e do concreto, da umidade da madeira, da temperatura, do tipo e das propriedades do elemento de ligação e das propriedades geométricas dos elementos acoplados. Em razão das propriedades da madeira, STOJIC \& CVETKOVIC (2006) advertem que as ligações por pinos não devem ser consideradas rígidas. Ressalta-se, nesta revisão, que a falta de normas específicas para tais ensaios tem dificultado o confronto de diversos valores de módulos de deslizamentos apresentados na literatura.

Como a rigidez da ligação é também dependente das direções dos conectores, o uso de elementos metálicos inclinados em determinadas situações melhora o comportamento da ligação, uma vez que cada conector passa a ser solicitado à tração, enquanto que, nos conectores dispostos verticalmente (Figura 2B), predominam as solicitações por flexão (JUTILA et al., 1997; KAVALIAUSKAS et al., 2007). Segundo relatam STEINBERG et al. (2003), no caso de parafusos inclinados, a rigidez da ligação pode até ser duplicada, em relação aos conectores instalados verticalmente. Tais resultados despertam a atenção para o efeito de embutimento da madeira, cuja resistência é relativa à compressão paralela às fibras, sendo o esmagamento das fibras mais crítico nas madeiras de baixa densidade.

O mecanismo de transferência de esforços por meio de conectores metálicos requer comprimentos de ancoragem na madeira e no concreto, sugeridos por GELFI et al. (2002), iguais a cinco e três vezes o diâmetro do conector, respectivamente. Frente às diversas situações expostas, é conveniente que tal informação seja verificada em laboratório para as condições de cada projeto, obtendo-se para cada material um valor 

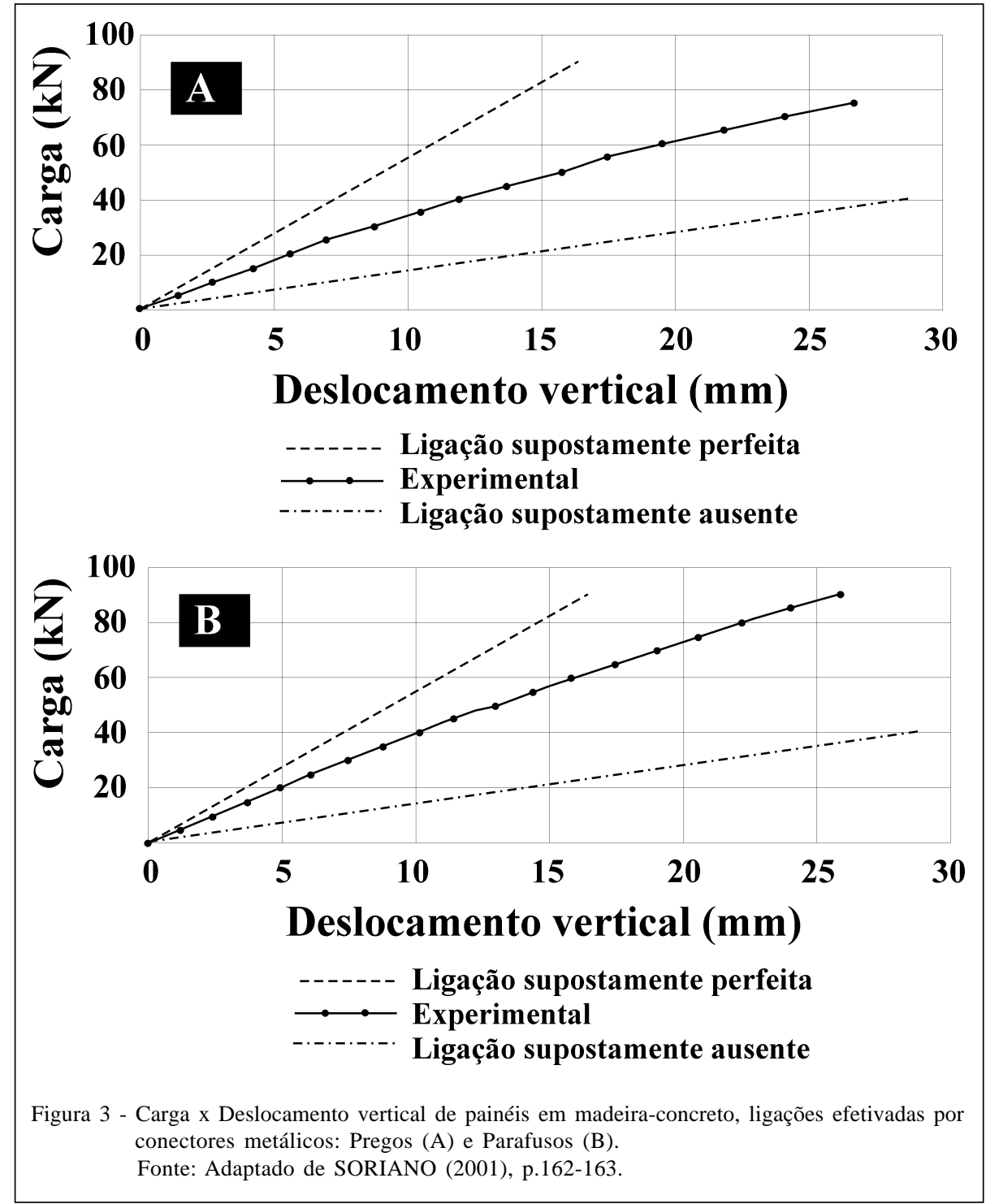

ideal de comprimento de ancoragem. Por um lado, valores aquém do ideal não asseguram a ancoragem, por outro, valores maiores não aumentam a eficiência da ligação. Diversos aspectos podem interferir na qualidade da ancoragem de barras de aço fixadas na madeira com adesivo epóxi, dentre os quais: preenchimento completo do vazio entre a barra e a parede do furo; a presença de extrativos oleosos na madeira; formação de bolhas de ar na resina, conforme adverte PIGOZZO (2004).

O sistema de ligação rígida pode ser estabelecido por meio de adesivo epóxi aplicado em toda interface de contato entre concreto e madeira (GIRHAMMAR \& GOPU, 1993). No entanto, BRUNNER et al. (2007) advertem que muitos cuidados são necessários, dentre os quais, destacam-se: observância do pot-life (tempo decorrido entre mistura do adesivo, aplicação e contato dos materiais), retração do concreto, deslocamento do adesivo durante a aplicação do concreto e temperatura. Adverte-se para esse sistema a possibilidade de ruptura frágil da estrutura, conforme verificado em ensaios realizados por SORIANO (2001). Além disso, a necessidade do controle rigoroso pode dificultar a aplicação do adesivo em campo.

O sistema de ligação formado por chapas metálicas coladas em fendas construídas na direção longitudinal das vigas de madeira foi pesquisado por CLOUSTON et al. (2005). Conforme relatado, o sistema apresentou rigidez efetiva à flexão 3\% menor que em uma viga com composição total. Frente às ligações discutidas anteriormente, tem-se para esta última a 
ligação de maior eficiência, cabendo, portanto, verificações para o uso em estruturas de pontes.

Modelagem das estruturas mistas em madeira-concreto

Os métodos de cálculo para estruturas mistas devem atender às condições dos Estados Limites, por exemplo, conforme procedimentos analíticos apresentados por CECCOTTI (1995). Conforme FRAGIACOMO \& CECCOTTI (2006), para as vigas de vãos médios a grandes ou em condições ambientais mais rigorosas, como no caso das pontes, o Estado Limite de Serviço de deslocamentos deve ser visto com grande atenção na elaboração de projetos, incluindose os efeitos de carregamento de longa duração. A modelagem computacional por elementos finitos, conforme apresentada por FRAGIACOMO \& CECCOTTI (2006), FRAGIACOMO (2006) e DIAS et al. (2007b), tem auxiliado no sentido de se realizar simulações para as estruturas mistas. Mesmo com tal ferramenta, levando-se em conta as variáveis envolvidas nesse sistema estrutural, a modelagem da ligação ainda representa um grande desafio na engenharia estrutural.

Apesar das várias incertezas de propriedades dos materiais e das condições ambientais, um bom ajuste entre os resultados da modelagem e o resultado experimental foi obtido por FRAGIACOMO \& CECCOTTI (2006), os quais empregaram o modelo viscoelástico para o concreto, com deformações inelásticas devidas à retração e à variação térmica. A simulação do comportamento mecânico das ligações com modelos não-lineares para os três materiais foi utilizada por DIAS et al. (2007 b), considerando, para tanto, o concreto e o aço isotrópicos e a madeira ortotrópica. As interações entre madeira-concreto, madeira-aço e concreto-aço foram simuladas por elementos de contato com atrito. Os resultados com valores superestimados foram, em parte, atribuídos ao modelo adotado para simular o comportamento da madeira, que deve ser melhorado para uma modelagem mais precisa, de acordo com DIAS et al. (2007b).

No atual estado da arte das estruturas mistas em madeira-concreto, evidenciam-se as preocupações mais recentes voltadas à interpretação do colapso em associação com os efeitos reológicos e as variações de temperatura, os quais devem ser associados à situação de carregamento mantido por longo tempo (KAVALIAUSKAS et al., 2005; CECCOTTI et al., 2007). Deve-se lembrar que o comportamento global da estrutura está condicionado ao comportamento de cada parte (madeira, concreto e ligação) submetida aos efeitos descritos, resultando em respostas diferenciadas e que associadas ao tempo ampliam o deslizamento da ligação e os deslocamentos na estrutura. Com isso, são alterados os esforços da estrutura.

O modo de falha de uma ligação é influenciado pelo módulo de elasticidade e resistência do concreto. Portanto, a escolha do concreto para formar a estrutura mista deve ser fundamentada, dentre outras razões, na natureza da estrutura. Segundo STEINBERG et al. (2003), o concreto de baixa densidade tem importância para as pequenas construções, principalmente nas reformas, graças aos menores acréscimos nas ações permanentes. Por outro lado, um concreto de alta resistência proporciona uma ligação com melhor desempenho e uma placa de menor espessura, conforme observaram DIAS et al. (2007a), ao ensaiarem ligações com concretos com agregados do tipo leve e normal e concreto de alta resistência (com resistência à compressão da ordem de 27, 46 e $84 \mathrm{MPa}$, respectivamente). Assim, pode-se concluir que os concretos de resistências mais elevadas são mais adequados para as construções de pontes que são expostas às condições mais agressivas, valendo-se, inclusive, do concreto de alta resistência, o qual deve proporcionar em sua seção uma ancoragem mais adequada para o conector metálico, além de assegurar maior durabilidade.

Considerando-se os conceitos discutidos nesta revisão sobre estruturas mistas em madeiraconcreto, bem como a necessidade de novas investigações e o vasto campo de aplicações desse sistema para as construções rurais, nota-se que o assunto não está esgotado. Confirma-se a viabilidade do emprego dessa técnica de estruturas mistas, uma vez que várias premissas de racionalização são atendidas, como, por exemplo, o uso adequado dos materiais, a exeqüibilidade e o baixo custo, como descrevem JUTILA et. al. (1997) e STOJIC \& KAJGANOVIC (2007). Acrescenta-se a esse princípio, conforme LEE (1999), a possibilidade, por exemplo, de, no mínimo, duplicar a vida útil de uma ponte que fosse construída somente em madeira.

Pontes mistas em madeira-concreto construídas no Brasil

As pontes destinadas às Classes 45 e 30, recentemente construídas no Brasil, com vãos da ordem de 15 e 7 metros (Figuras 4A e 4B, respectivamente), ilustram aplicações da técnica de estrutura mista em madeira e concreto. A instalação dos conectores metálicos com adesivo epóxi nas longarinas de Eucalipto citriodora tratado pode ser vista na figura 4C, e a aplicação de concreto usinado sobre o tabuleiro

Ciência Rural, v.39, n.4, jul, 2009. 

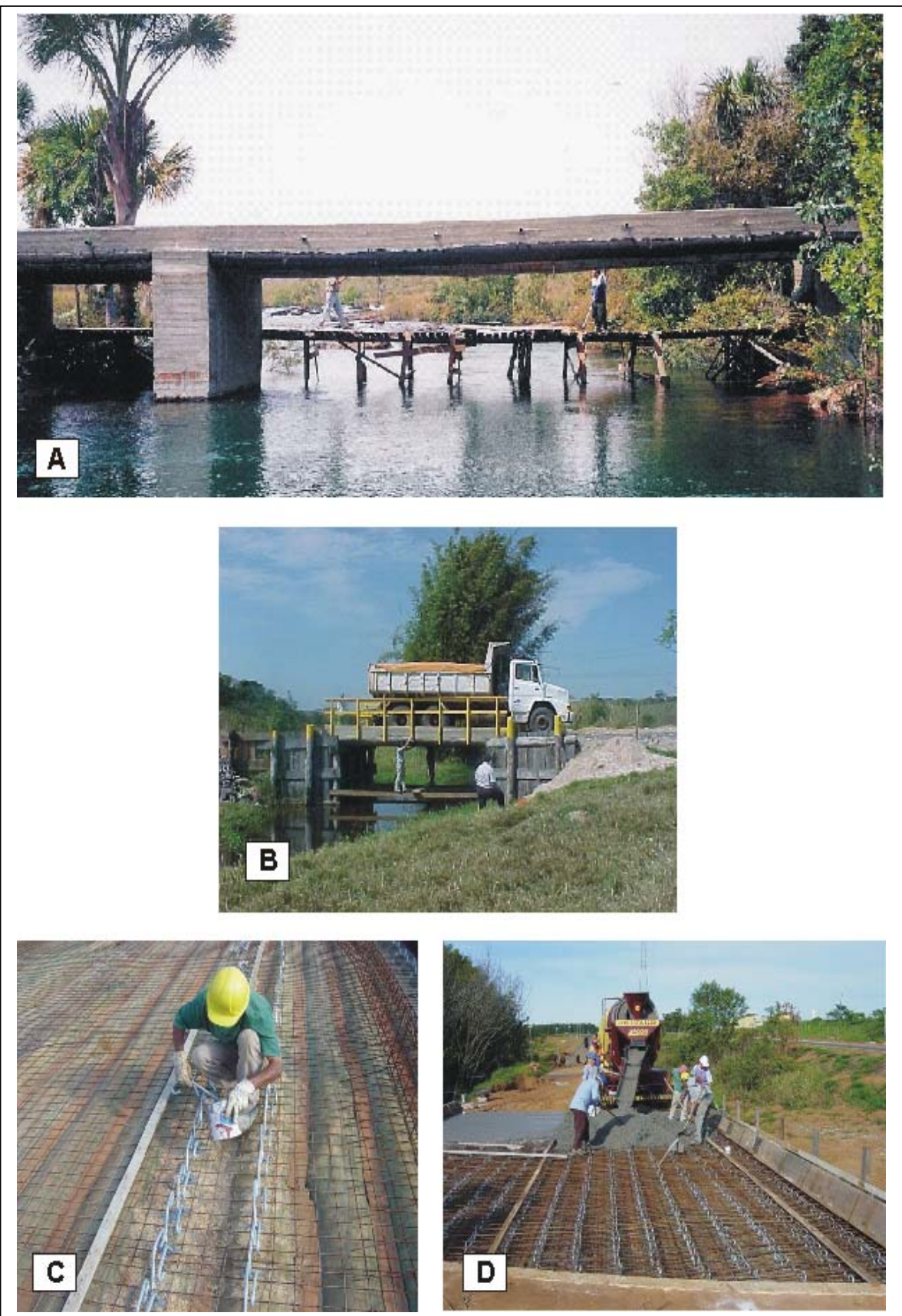

Figura 4 - Pontes mistas em madeira-concreto com largura de 4,0m; longarinas roliças em eucalipto citriodora: Comprimento total de 20,45m; fundações em concreto armado (A). Comprimento total de 7,00m; fundações em estacas de madeira (B). Detalhes de execução: Fixação dos conectores com adesivo epóxi (C) e Concretagem do tabuleiro (D).

Fonte: CALIL JÚNIOR et al. (2006), p. A4, A13 e A25.

é representada na figura 4D. Destaca-se que as peças de madeira, que, em parte, compõem a estrutura do tabuleiro também constituem parte da fôrma para concretagem, dispensando, inclusive, o uso de escoramentos. Finalmente, a técnica estrutura mista em madeira-concreto proporciona uma solução viável para as construções novas ou as reformas de pontes, o que contribui para a melhoria da malha de estradas vicinais, favorecendo, assim, a integração regional e o escoamento da produção agrícola.

Ciência Rural, v.39, n.4, jul, 2009. 


\section{CONCLUSÕES}

Dentre os sistemas estruturais para a construção de pontes de estradas vicinais, destaca-se a técnica de estrutura mista em madeira e concreto, que proporciona uma solução racional para o uso desses materiais, resultando em benefícios para o desempenho e a durabilidade da construção. O uso de conectores metálicos tem apresentado resultados satisfatórios para a efetivação do sistema de ligação, transmitindo, assim, os esforços na interface de contato entre os dois materiais.

A simulação das estruturas mistas apresenta complexidade inerente ao uso de materiais com propriedades distintas, as quais devem ser relacionadas aos efeitos de variações térmicas e de carregamento mantido ao longo do tempo. No entanto, resultados teórico-experimentais demonstram a importância e viabilidade dessa técnica para construção de pontes das estradas vicinais.

\section{REFERÊNCIAS}

AHMADI, B.H.; SAKA, M.P. Behavior of composite timberconcrete floors. Journal of Structural Engineering, New York, v.119, n.10, p.3111-3130, 1993.

ASSOCIAÇÃO BRASILEIRA DE NORMAS TÉCNICAS. Projeto de estruturas de concreto - procedimento: NBR 6118. Rio de Janeiro, 2003. 221p.

BRUNNER, M. et al. Behavior of composite timber-concrete floors. Materials and Structures, Bagneux, v.40, n.1, p.119126, 2007.

CALIL JÚNIOR, C. et al. Manual de projeto e construção de pontes de madeira. São Carlos: Suprema, 2006. 252p.

CARVALHO, R.C.; FIGUEIREDO FILHO, J.R. Cálculo e detalhamento de estruturas usuais de concreto armado: segundo a NBR 6618/2003. São Carlos: EdUFSCar, 2004. 374p.

CECCOTTI, A. Timber-concrete composite structures: timber engineering - STEP1/Eurofortech lecture series. Almere: BLASS, H.J. et al., 1995. E13, p.1-12.

CECCOTTI, A. et al. Long-term and collapse tests on a timberconcrete composite beam with glued-in connection. Materials and Structures, Bagneux, v.40, n.1, p.15-25, 2007.

CLOUSTON, P. et al. Shear and Bending performance of a novel wood-concrete composite system. Journal of Structural Engineering, New York, v.131, n.9, p.1404-1412, 2005.

DIAS, A.M.P.G.. et al. Load-carrying capacity of timberconcrete joints with dowel-type fasteners. Journal of Structural Engineering, New York, v.133, n.5, p.720-727, 2007a.
DIAS, A.M.P.G.. et al. A non-linear 3D FEM model to simulate timber-concrete joints. Advances in Engineering Software, Oxford, v.38, p.522-530, 2007b. Disponível em: http:// $\mathrm{w}$ w $\mathrm{w}$. $\mathrm{s}$ c i e $\mathrm{n}$ c e d i r e c t c o m / science?_ob=ArticleURL\&_udi=B6V1P-4MSR8KM-

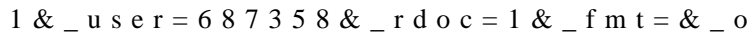
rig=search \&_sort $=$ d \&view $=$ c $\& \_$acct $=C 000037899 \& \_v e r s i o n=$ 18 _urlVersion $=0$ \&_userid=687358\&md5=a6c4e3097dc63087fda41a8f55659b7c. Doi: c10.1016/j.advengsoft.2006.08.024.

FONTE, T.F.; CALIL JÚNIOR, C. Pontes protendidas de madeira: alternativa técnico-econômica para vias rurais. Engenharia Agrícola, Jaboticabal, v.27, n.2, p.552-559, 2007.

FRAGIACOMO, M.; CECCOTTI, A. Long-term behavior of timber-concrete composite beams. I: Finite Element modeling and validation. Journal of Structural Engineering, New York, v.132, n.1, p.1-12, 2006.

FRAGIACOMO. M. Long-term behavior of timber-concrete composite beams. II: Numerical analysis and simplified evaluation. Journal of Structural Engineering, New York, v.132, n.1, p.13-22, 2006.

FRAGIACOMO, M. et al. Short- and long-term performance of the "Tecnaria" stud connector for timber-concrete composite beams. Materials and Structures, Bagneux, v.40, p.10131026, 2007. Disponível em: http://www.springerlink.com/ content/71557p4144n85172/. Doi: 10.1617/s11527-0069200-2.

GELFI, P. et al. Stud shear connection design for composite concrete slab and wood beams. Journal of Structural Engineering, New York, v.128, n.5, p.1544-1550, 2002.

GIRHAMMAR, U.A.; GOPU, V.K.A. Composite beam-columns with interlayer slip-exact analysis. Journal of Structural Engineering, New York, v.119, n.4, p.1265-1282, 1993.

GUTKOWSKI, R.M. et al. Investigation of notched composite wood-Concrete connections. Journal of Structural Engineering, New York, v.130, n.10, p.1553-1561, 2004.

JUTILA, A. et al. Testing a wood-concrete composite bridge. Structural Engineering International, Yvelines, v.7, n.4, p.275-277, 1997. Disponível em: http://www.ingentaconnect.com/ content/iabse / s e i / $1997 / 00000007 / 00000004$ / art00017?token=005214ea593 25a666f3a7b2f7c40386f3b6b46 52586b4649264f655d3 75c6b6876305021d798624929. Doi: $10.2749 / 101686697780494635$.

KAVALIAUSKAS, S. et al. Evaluation of long-term behaviour of composite Timber-concrete structures according to EC. Journal of Civil Engineering and Management, Vilnius, v.11, n.4, p.292-296, 2005.

KAVALIAUSKAS, S. et al. Mechanical behaviour of timberto-concrete connections with inclined screws. Journal of Civil Engineering and Management, Vilnius, v.13, n.3, p.193-199, 2007.

LEE, A.G.. Bridge option for forest roads: a composite timber beam and concrete deck bridge Forestry Tasmania, 1999. Acessado em 7 jan. 2008. Online. Disponível em: http://www.forestrytas.com.au/assets/0000/0173/ tasforests11_part_6.pdf.

Ciência Rural, v.39, n.4, jul, 2009. 
MASCIA, N.T.; SORIANO, J. Benefits of timber-concrete composite action in rural bridges. Materials and Structures, Bagneux, v.37, n.2, p.122-128, 2004. Disponível em: https:// $\mathrm{w}$ w w $\mathrm{r}$ i l e m . n e t / b o u t i q u e fiche.php?cat=journal\&reference=1322. Doi: 10.1617/13822.

MIOTTO, J.L.; DIAS, A.A. Reforço e recuperação de estruturas de madeira. Semina: Ciências Exatas e Tecnológicas, Londrina, v.27, n. 2, p.163-174, 2006.

PIGOZZO, J.C. Estudo e aplicações de barras de aço coladas, como conectores em lajes mistas de madeira e concreto para tabuleiros de pontes. 2004. 358f. Tese (Doutorado em Engenharia de Estruturas) - Curso de Pósgraduação em Engenharia Civil. Universidade de São PauloEESC.

SEGUNDINHO, P.G..A.; MATTHIESEN, J.A. Análise experimental de um modelo reduzido de uma ponte mista de madeira-concreto utilizando vigas de madeira roliça. In: ENCONTRO BRASILEIRO EM MADEIRAS E EM ESTRUTURAS DE MADEIRA, 9., 2004, Cuiabá, MT. Anais.. Cuiabá : UFMT, 2004. 1 CD.

SORIANO, J. Estruturas mistas em concreto e em madeira: Análise de vigas e painéis e aplicações na construção civil. 2001. 264f. Tese (Doutorado em Construções Rurais e Ambiência) - Curso de Pós-graduação em Engenharia Agrícola, Universidade Estadual de Campinas.

SOUZA, V.C.M.; RIPPER, T. Patologia, recuperação reforço de estruturas de concreto. São Paulo: Pini, 2004. 255p.

STEINBERG, E. et al. Connectors for timber-lightweight concrete composite structures. Journal of Structural Engineering, New York, v.129, n.11, p.1538-1545, 2003.

STOJIC, D.; CVETKOVIC, R. Analysis of a composite timberconcrete structures according to the limit states Design and innovative methods in coupling of a timber and concrete. Facta Universitatis, Series: Architecture and Civil Engineering, Nis, v.2, n.3, p.169-184, 2001.

STOJIC, D.; CVETKOVIC, R. Design of connections in composite timber-concrete structures. Facta Universitatis, Series: Architecture and Civil Engineering, Nis, v.4, n.2, p.127-138, 2006.

STOJIC, D.; KAJGANOVIC, T. Composite timber-concrete road bridge structure. Facta Universitatis, Series: Architecture and Civil Engineering, Nis, v.5, n.2, p.141148, 2007. 